Revue d'histoire de l'Amérique française

REVUE D.HISTOIRE DE L'AMÉRIQUE FRANÇAISE

\title{
Les classes dominantes au Québec, 1760-1840. Bilan historiographique
}

\section{Fernand Ouellet}

Volume 38, numéro 2, automne 1984

Bourgeoisies et Petites Bourgeoisies

URI : https://id.erudit.org/iderudit/304261ar

DOI : https://doi.org/10.7202/304261ar

Aller au sommaire du numéro

Éditeur(s)

Institut d'histoire de l'Amérique française

ISSN

0035-2357 (imprimé)

1492-1383 (numérique)

Découvrir la revue

Citer cet article

Ouellet, F. (1984). Les classes dominantes au Québec, 1760-1840. Bilan historiographique. Revue d'histoire de l'Amérique française, 38(2), 223-243.

https://doi.org/10.7202/304261ar d'utilisation que vous pouvez consulter en ligne.

https://apropos.erudit.org/fr/usagers/politique-dutilisation/ 


\title{
LES CLASSES DOMINANTES AU QUÉBEC, 1760-1840. BILAN HISTORIOGRAPHIQUE
}

\author{
FERNAND OUELLET \\ Département d' histoire \\ Université d'Ottawa
}

C'est, d'habitude, dans les moments où l'historiographie est en voie de se transformer rapidement que les historiens ont davantage tendance à s'interroger à haute voix et par écrit sur leur métier, pour ensuite se reposer plus ou moins longtemps sur des certitudes méthodologiques. Ainsi, au commencement du $\mathrm{XX}^{\mathrm{e}}$ siècle, au moment où débute la professionnalisation de la discipline au Canada anglais, ce fait est particulièrement bien illustré par les Mémoires de la Société royale du Canada, dont certains textes, très volumineux, marquent une forte influence des positivistes allemands ${ }^{1}$. Plus tard, alors que s'engage la diversification de cette historiographie anglophone durant les années 1930, non seulement ce genre d'écrits se fit-il plus fréquent mais il devint plus ou moins de tradition à la Société historique du Canada que, chaque année, le président sortant donnât de préférence à son discours, si ce n'est une tournure franchement historiographique, au moins une certaine coloration philosophique ${ }^{2}$. Il en fut de même au Québec, vers 1950, alors que les départements d'histoire de Laval et de Montréal venaient d'être fondés et que s'engagea parmi les francophones le même mouvement de professionnalisation qu'au Canada anglais: en fait, presque tous les articles, publiés par Marcel Trudel sur la signification de la discipline, à l'exception de son discours présidentiel à la Société historique du Canada en 1966 et de son propos d'adieu en 1982, affichèrent ces préoccupations ${ }^{3}$.

Mais, depuis 1960 en particulier, ces façons de se poser, ouvertement et avec plus ou moins d'ardeur au combat, des questions à propos de la discipline font partie de notre existence, reflet sans aucun doute d'une société en voie de se transformer d'une manière accélérée et d'une historiographie, chez nous et ailleurs dans le monde, en perpétuel changement. La pratique des colloques et des rencontres plus ou

\footnotetext{
$1 \quad$ F. Ouellet, «L'émergence dans le Canada du $\mathrm{XX}^{\mathrm{e}}$ siècle de l'histoire comme science sociale», dans MSRC (1982): 46; voir MSRC (1911): 127-179; (1912): 133-164; (1915): 489587; (1919): 9-19; (1921): 1-15; (1930): 63-91; (1933): 8; (1936): 1-8.

2 Ibid.: 51 . 1978), 8-13.

Voir Mélanges d'histoire du Canada français offerts à Marcel Trudel (Ottawa, EUO,
} 
moins formels, où les historiens se demandent où ils en sont et où ils vont, est devenue incessante. Leurs thèmes varient au gré d'une pratique historienne, qui colle d'assez près au présent et qui, par conséquent, est directement touchée (mais sans être exceptionnelle à cet égard parmi les sciences sociales) par les mutations sociales, les brassages idéologiques et par le développement des méthodes et des techniques scientifiques dans les sciences de l'homme.

En tout cas, depuis une vingtaine d'années, les historiens qui pratiquent l'historiographie, la méthodologie et l'épistémologie et ceux qui eurent quelque chose de vibrant à dire dans leur domaine de spécialisation, n'ont pas chômé. Régulièrement, ils furent mobilisés pour procéder à des bilans et à des perspectives ou, pour dire les choses d'une façon plus sophistiquée, pour se livrer à des anamorphoses et à des prospectives $^{4}$. Pour ma part, depuis 1962, en comptant les seules participations de ce genre qui aboutirent à des publications, je peux aligner sous ma signature une dizaine de textes ${ }^{5}$. Je pourrais presque en dire autant de mes collègues Wallot et Paquet qui, pourtant, entrèrent dans la carrière plus tard que moi ${ }^{6}$. Je devrais mentionner plusieurs autres historiens qui se trouvent dans la même situation. Mais, si je me contente pour le moment de ne mentionner que ces noms familiers, c'est que, au total, plus que d'autres ils furent mêlés à ces exercices de diagnostic et à des débats qui en disent long sur le climat qui a entouré la transformation de l'historiographie dans notre milieu.

Cela étant dit, il faut souligner que le présent texte se démarque d'un courant de plus en plus affirmé dans notre historiographie. En effet, au Québec, comme dans le reste du Canada, l'intérêt des étudiants et des chercheurs s'est déplacé d'une façon radicale vers le contemporain et l'actuel. Un relevé statistique des articles parus dans trois publications canadiennes: les Mémoires de la Société royale du Canada, la Canadian Historical Review et la Revue d'histoire de

\footnotetext{
4 G. Paquet et J.-P. Wallot, «Anamorphoses et prospectives», dans Économie québécoise (Montréal, PUM, 1969), 255-301.

5 Voici quelques titres: "L'Étude du XIX $\mathrm{X}^{\mathrm{e}}$ siècle canadien-français», dans Recherches sociographiques (1962): 27-42; «Histoire et sociologie: le point de vue de l'historien», dans le RASHC (1966): 166-177; «L'histoire sociale du Bas-Canada. Bilan et perspectives de recherches», dans RASHC (1970): 1-18; «La recherche historique au Canada français», dans L. Beaudoin (éd.), La Recherche au Canada français (Montréal, PUM, 1969), 87-98; «Historiographie canadienne et nationalisme», dans MSRC (1975): 25-39; «L'histoire socio-culturelle: colloque exploratoire», dans Histoire sociale/Social History (1976): 5-10; «Quebec, 1760-1867» in D.A. Muise (ed.), A Reader's Guide to Canadian History, 1: Beginnings to Confederation (Toronto, UTP, 1982), 45 77; «La philosophie de l'histoire et la pratique historienne d'hier et d'aujourd'hui», dans D. Carr, W. Dray, T. Geraets, F. Ouellet, H. Watelet, (ed.), La philosophie de l'histoire et la pratique historienne d'aujourd' hui (Ottawa, EUO, 1982), 215-235.

6 En plus de "Anarnorphoses et prospectives», voir G. Paquet et J.-P. Wallot, «Le BasCanada au début du XIX ${ }^{\mathrm{e}}$ siècle: une hypothèse», dans RHAF (1971): 39-63; «Pour une mésohistoire du XIX ${ }^{\mathrm{e}}$ siècle canadien-français», dans RHAF (1979): 387-427; "Sur quelques discontinuités dans l'expérience socio-économique du Québec», dans RHAF (1982): 483-523.
} 
l'Amérique française ${ }^{7}$, le prouve comme il démontre un recul équivalent de l'attrait pour la Nouvelle-France et, d'une façon générale, pour la période pré-confédérative. Cette conclusion est d'ailleurs confirmée par un calcul rapide de l'activité de recherche, telle qu'elle apparaît à partir du Répertoire des thèses en cours pour l'année 1981: seulement $12,8 \%$ des thèses portant sur le Québec concernent le Québec des années 1760 à 1840 et seulement 7,6\% d'entre elles s'adressent, directement ou indirectement, à la question des classes dominantes: noblesse, clergé et bourgeoisie ${ }^{8}$.

Si cette historiographie des années 1760-1840 a une signification particulière par rapport à celle des autres périodes, c'est plutôt parce que, à mon avis, les débats historiographiques qui ont eu lieu depuis le milieu des années 1950, dans leurs rapports avec le développement de la société québécoise, ont contribué jusqu'à un certain point à façonner les perspectives qui ont servi à encadrer la recherche, qu'il s'agisse de la Nouvelle-France, du siècle qui suit la conquête ou des années ultérieures. Il va sans dire que cela inclut non seulement la recherche sur les classes dominantes mais également celle sur les classes dominées. Parmi ces débats qui se relient les uns aux autres mais qui se rattachent à des orientations spécifiques de l'historiographie, je voudrais commenter le débat sur la conquête et la bourgeoisie, celui sur la crise agricole et un troisième sur les insurrections de 1837-38.

\section{Le débat sur la conquête: une confrontation entre le national et le social.}

Le débat sur la conquête ${ }^{9}$ s'est engagé vers le milieu des années 1950 et il s'est poursuivi pendant une dizaine d'années pour ensuite perdre peu à peu de son intensité. Incontestablement, il était le résultat des prises de conscience qui s'étaient amorcées au lendemain du second conflit mondial dans les nouvelles couches de la bourgeoisie canadiennefrançaise et, surtout, parmi les intellectuels; les unes conduisaient à l'émergence de la pensée néo-nationaliste, les autres à une remise en question du pouvoir des classes dirigeantes traditionnelles dont le nationalisme clérical et conservateur était l'idéologie dominante. À tous ces courants devaient finalement correspondre des visions différentes du type de société qui avait existé dans le passé jusqu'à l'époque de la Nouvelle-France. En l'occurrence, dans la perspective de l'évaluation

\footnotetext{
7 «L'émergence dans le Canada du XXe siècle de l'histoire comme science sociale», dans MSRC (1982): 73-81; voir à propos de la RHAF, F. Harvey et P.-A. Linteau, «L'évolution de l'historiographie dans la RHAF, 1947-1972», dans RHAF (1972): 163-183; J.-P. Coupal, «Les dix dernières années de RHAF, 1972-1981», dans RHAF (1983): 553-569.

8 Répertoire des thèses en cours portant sur des sujets d' histoire et autres sujets connexes (SHC, Ottawa, 1981), $100 \mathrm{p}$.

Les deux textes suivants marquent le début du débat: F. Ouellet, «Michel Brunet et le problème de la conquête», dans BRH (1956): 92-101; compte rendu G. Frégault, La guerre de la conquête, dans Vie des Arts (1956): 33s.
} 
des conséquences de la conquête, les concepts de société traditionnelle, de société d'Ancien Régime et de «société bourgeoise» appliqués à la Nouvelle-France prirent une importance capitale. Même l'idée de décapitation sociale qui remontait à F.-X. Garneau n'était pas encore un bon indicateur à cet égard puisque jusqu'à Maurice Séguin, personne parmi les historiens canadiens-français n'était allé jusqu'à soutenir qu'elle s'était exercée sur la classe qui portait en elle le devenir même de la nation.

Lorsqu'en 1946, Maurice Séguin ${ }^{10}$ avait esquissé les premiers éléments de base de l'interprétation néo-nationaliste, prévalait alors chez la majorité des historiens canadiens-français dont Groulx était le chef de file, l'idée que, en Nouvelle-France, l'économie était agricole et la société théocratique ${ }^{11}$. Dans ces conditions, le phénomène de la décapitation sociale ne pouvait être bien tragique puisqu'il laissait intacte la seule élite indispensable à la survie de la nation: le clergé. On ne trouve pas davantage, dans le livre de G. Frégault, La civilisation de la Nouvelle-France (1944), de liens possibles entre la décapitation sociale et la gravité des répercussions de la conquête. En effet, Frégault, en plus de soutenir que l'économie était agricole, prétend non seulement qu'il n'y avait pas de bourgeoisie en Nouvelle-France mais que la société était dominée par une classe de seigneurs ecclésiastiques et laïques tirés en majorité du peuple ${ }^{12}$. Naturellement, la nation, encore au stade de la formation, devait l'essentiel de sa solidité et de sa vigueur à cette forme épurée d'Ancien Régime social qui permettait aux élites, disaiton, de vivre en harmonie avec la société qu'elles dominaient.

Fait surprenant, il existait un historien anglophone qui, avant Séguin, avait lié d'une façon essentielle les conséquences de la conquête au fait même de la décapitation sociale: c'est-à-dire à l'idée de l'élimination de la bourgeoisie. Il s'agissait de Donald Creighton ${ }^{13}$ qui, partant de la théorie des staples pour établir les bases économiques du développement de la nation canadienne, avait prétendu que l'économie de la Nouvelle-France était fondée sur le commerce des pelleteries et non sur l'agriculture et que la société était, en conséquence, dirigée par une bourgeoisie qui possédait en germe les caractéristiques d'une bourgeoisie nationale. Pour Creighton, cette bourgeoisie qui avait contribué à faire naître un embryon de conscience nationale parmi les habitants

10 «La conquête et la vie économique des Canadiens», dans L'Action nationale (1946), texte repris dans Économie québécoise (Montréal, PUM, 1969), 345-361 et dans R. Durocher et P.-A. Linteau, Le "Retard» du Québec et l' infériorité économique des Canadiens français (Boréal Express, 1971), 93-111.

11 Analysé dans F. Ouellet, «La formation d'une société dans la vallée du Saint-Laurent: d'une société sans classes à une société de classes», dans la CHR (1981): 413-421.

12 Ibid.: 421-423.

13 D.G. Creighton, The Commercial Empire of the St. Lawrence (Toronto, Macmillan, 1937), 441 p.; Dominion of the North. A History of Canada (Boston, Houghton Mifflin, 1944), $535 \mathrm{p}$. 
de la Nouvelle-France vivait en harmonie avec la société coloniale et avec la métropole qui inspirait son avenir. Mais le chef de l'École laurentienne avait aussi prétendu que la société locale possédait en plus un élément féodal lié à une faible agriculture de subsistance et, pour cette raison, appelé avec le temps à se dégrader, tout comme les classes dirigeantes qui en dépendaient. C'est l'acceptation par Creighton du fait de la décapitation sociale et du remplacement de la bourgeoisie francophone par une bourgeoisie anglophone qui l'amène, d'une part, à affirmer que la conquête n'avait rien changé de fondamental aux structures sociales et, d'autre part, à décrire la société canadiennefrançaise de l'après-conquête comme une société semi-féodale décadente devenue hostile aux impératifs économiques.

Ce schéma interprétatif est-il bien différent de celui qui fut proposé plus tard par Maurice Séguin, par Guy Frégault après son adhésion au néo-nationalisme et par Michel Brunet ${ }^{14}$ ? Bien sûr, à lire les uns et les autres, on voit immédiatement les différences; mais il n'en reste pas moins que tous ces auteurs étaient partis de présupposés assez semblables à propos de la nation et de son développement et qu'ils arrivèrent à des conclusions assez similaires quant à la nature du processus historique. En effet, des travaux de Séguin, de Frégault et de Brunet se dégage aussi l'image d'une colonie qui, avant 1760, se développe tellement en harmonie avec sa métropole dite nourricière que les rapports de dépendance qui existent entre elles paraissent si inoffensifs que ses habitants, en particulier les bourgeois, semblent à peu près complètement maîtres de leur destin et de celui de la nation qui est en train de se constituer sous l'oeil complice de la mère patrie. Comme l'économie coloniale repose avant tout sur le commerce des pelleteries, le leadership de la société appartient naturellement aux éléments hétérogènes qui, selon eux, composent la bourgeoisie: les négociants, les traiteurs, les nobles, les militaires et les administrateurs. En somme, cette bourgeoisie originale qui vit tellement près de son milieu, occupe presque toute la place au sommet de la société sans même subir la tutelle de la bourgeoisie de la métropole, ce qui pourrait paraître inquiétant pour celui qui croit aux rapports habituels et nécessaires de dépendance entre le centre et la périphérie. Ainsi le caractère dramatique de la conquête dépend essentiellement de la déchéance subséquente de cette catégorie sociale à la suite de la victoire militaire des Britanniques et de leur entrée en possession du pouvoir politique. L'histoire des classes dominantes après 1760 , d'après les premiers néo-nationalistes, serait

14 M. Séguin, L'idée d'indépendance au Québec (Trois-Rivières, Boréal Express, 1969); G. Frégault, La guerre de la conquête (Montréal, Fides, 1955), 514 p.; M. Brunet, La présence anglaise et les Canadiens (Montréal, Beauchemin, 1964), 293 p.; Canadians et Canadiens: études sur l'histoire et la pensée des deux Canadas (Montréal, Fides, 1954), 173 p.; Les Canadiens après la conquête, 1759-1775: de la révolution canadienne à la révolution américaine (Montréal, Fides, 1969). 
donc celle du passage de la bourgeoisie, classe dominante, au rang de petite bourgeoisie sans influence et sans pouvoir, dominée au surcroît par la bourgeoisie anglophone, et celle de la remontée du pouvoir du clergé, maintenant coincé entre le conquérant et le peuple.

Cette conception de la société de la Nouvelle-France, qui suppose tellement de bonne volonté de la part des partenaires sociaux et politiques et qui, pratiquement, exemptait les habitants de la colonie des luttes que les autres colonies devaient mener pour conquérir leur indépendance et transformer leur société, ne nous a jamais convaincu. À mesure que notre recherche progressait, nous avons toujours eu tendance à voir dans la société coloniale de l'époque française une société d'Ancien Régime au même titre que celle de la métropole ${ }^{15}$. Pour nous, la Nouvelle-France était dominée par une noblesse militaire et foncière, avantagée dans le commerce des fourrures et jouissant de très forts appuis politiques, et par un clergé étroitement uni à l'État et privilégié. Quant à la bourgeoisie, attirée comme elle l'était par la noblesse, elle dépendait aussi bien des nobles que de l'État. Comme l'indique le livre de J. Hamelin, Économie et société en Nouvelle-France, les bourgeois de la colonie dépendaient tout autant des entreprises et des marchands de la métropole et de leurs représentants, de sorte que, au jour le jour, ils devaient payer un tribut à la métropole pour la protection militaire qu'elle accordait à l'économie des pelleteries et les avantages qu'elle consentait à celles-ci sur ses propres marchés. Comment, dans ces conditions, pouvait-on reconnaître à cette bourgeoisie coloniale si dépendante et si faible un rôle quelconque dans l'éclosion prochaine d'une conscience nationale? C'est parce que nous avons pu suivre de la conquête jusqu'en 1850 l'évolution respective de la noblesse, du clergé et de la petite bourgeoisie née avant 1760, enrichie des professions libérales au cours de la seconde moitié du siècle, que nous avons rejeté la thèse de la décapitation sociale et attribué le distancement des francophones dans le dornaine économique moins à des facteurs externes qu'à des facteurs internes d'ordre socio-culturel. À l'époque de la publication de notre Histoire économique et sociale, nous eûmes tendance à insister sur la survivance presque intégrale au XVIII siècle de la structure sociale héritée de l'époque française. Mais, depuis ce temps, notre point de vue s'est modifié en ce qui regarde les conséquences négatives de la conquête sur le rôle politique et militaire de la noblesse. C'est aussi parce que les institutions sociales de la colonie n'étaient pas dans

15 F. Ouellet, Histoire économique et sociale du Québec, 1760-1850. Structures et conjoncture (Montréal, Fides, 1966), 640 p.; Le Bas-Canada. Changements structuraux et crise (Montréal, EUO, 1976), 539 p.; «Propriété seigneuriale et groupes sociaux dans la vallée du SaintLaurent (1663-1840)», dans Mélanges d' histoire du Canada offerts au professeur Marcel Trudel (Ottawa, EUO, 1978), 183-213; «Officiers de milice et structure sociale au Québec (1660-1815)», Histoire sociale (1979): 37-65; «Dualité économique et changement technologique au Québec, 1760-1790», dans Histoire sociale (1976): 256-296; «Libéré ou exploité! Le paysan québécois d'avant 1850», dans Hisioire sociale (1980): 339-368. 
la pratique substantiellement différentes de celles de la France que nous avons pu voir émerger à long terme un conflit entre le seigneurialisme et le capitalisme, même sous sa forme commerciale.

$\mathrm{Au}$ fond, ces deux schémas interprétatifs recouvrent deux types principaux de préoccupations qui peuvent paraitre exclusives: l'une avec la question nationale et l'autre avec la question sociale, telle qu'elle pouvait se poser dans le contexte du besoin de libéralisation des années 1950-60 et, faut-il ajouter, à partir de notre propre fréquentation des historiens de l'École des Annales. La progression rapide après $1960 \mathrm{du}$ nationalisme indépendantiste, dans les classes moyennes comme dans les milieux intellectuels sur l'ensemble du territoire québécois, explique la vogue grandissante dans la recherche et l'enseignement de la perspective néo-nationaliste; celle-ci, cependant, devait se transformer jusqu'à un certain point avec la montée d'une sensibilité plus grande à la question sociale.

\section{Le débat sur la crise agricole: histoire et sciences de l'homme}

Jusqu'en 1970, l'histoire économique et sociale du Canada avait progressé si lentement qu'une fois éliminés de la liste des publications en ce domaine les noms de H. Innis, D.G. Creighton, A.R.M. Lower, F. Ouellet et quelques articles de Michel Brunet, on pouvait compter sur les doigts de la main les productions traitant des classes dirigeantes et, plus rarement encore, des milieux populaires. Tout au plus quelques livres plus ou moins reliés à ces sujets par M. Trudel ${ }^{16}$, J.-J. Jolois ${ }^{17}$ et Lucien Lemieux ${ }^{18}$ ainsi que quelques articles plus indispensables de L.Edmond Hamelin ${ }^{19}$ et de Louise Dechêne ${ }^{20}$ complètent à peu près ce maigre bilan. Pourtant, malgré cette pénurie plus apparente que réelle, on pouvait aisément avoir le sentiment qu'un changement était en cours. En effet, depuis les années 1950, le message de l'École des Annales en faveur d'une histoire économique et sociale orientée vers le quantitatif et ouverte sur les autres sciences sociales avait quand même été diffusé. Pour notre part, depuis 1957 au moins, alors que nous avions signé dans la RHAF le premier volet d'un long compte rendu de l'oeuvre magistrale et colossale de Pierre Chaunu sur Séville et l'Atlantique et que nous avions dans un court article tenté de tester à propos de la

\footnotetext{
16 M. Trudel, L'influence de Voltaire au Canada (Montréal, Fides, 1945), 2 vol.; L'Église canadienne sous le régime militaire (Montréal et Québec, 1956-57), 2 vol.; Chiniquy (TroisRivières, Bien Public, 1955); «La servitude de l'Église catholique du Canada français sous le régime anglais», RASHC (1963): 42-64.

17 J.-J. Jolois, J.-F. Perreault (1753-1844) et les origines de l' enseignement laïque au BasCanada (Montréal, PUM, 1969).

18 L. Lemieux, L'établissement de la première province ecclésiastique au Canada, 1783 1844 (Montréal, Fides, 1968).

19 L.-E. Hamelin, «Évolution numérique séculaire du clergé catholique dans le Québec», dans Recherches sociographiques (1961): 189-243.

20 L. Dechêne, «Les entreprises de William Price», dans Histoire sociale (1968): 16-53.
} 
pratique de l'encan un modèle de l'ethnologue français Marcel Mauss, nous avons orienté nos travaux en fonction des perspectives de l'École historique française ${ }^{21}$. En 1960, mon collègue, Jean Hamelin qui fit tant pour développer le travail d'équipe au Québec, avait publié son livre Économie et société en Nouvelle-France qui épousait étroitement la méthodologie des Annales ${ }^{22}$. Puis, est venu le colloque de Laval en 1963, auxquels participaient plusieurs professeurs français liés à ce courant, dont Labrousse, Mandrou et Dupront, où Hamelin et moimême avions présenté trois communications à caractère économique et social $^{23}$. C'est dire toute la signification que nous attachions à cette rencontre à laquelle furent présents et actifs nombre de collègues des sciences sociales et des géographes. La parution des Actes de ce colloque précéda de peu celle de notre Histoire économique et sociale, 1760-1850. Structures et conjoncture, livre qui, quelques années plus tard eut sa suite dans celui de Hamelin et Roby ${ }^{24}$. C'est aussi le moment où paraissait le texte d'Alfred Dubuc sur les structures sociales au Québec de 1760 à $1840^{25}$ dans lequel il exposait la thèse du pacte aristocratique et représentait le mouvement patriote comme une aventure progressiste. Le lancement de cette hypothèse marquait l'apparition parmi les historiens francophones d'une perspective marxiste qui constituait, en même temps, une autre incitation à l'intention des historiens à s'alimenter davantage dans les autres sciences de l'homme.

En fait, déjà, depuis le début des années 1950, avait existé parmi les praticiens des sciences sociales un intérêt très marqué pour les perspectives historiques. C'est un fait dont témoignent deux livres parus à cette époque: Les essais sur le Québec contemporain (1953), et La grève de l'amiante $(1956)^{26}$. Pour appuyer leurs remises en question du Québec traditionnel, les sociologues utilisaient le modèle de la Folk Society, opposaient la société traditionnelle et la société industrielle et, étaient, par conséquent, voués à un recours incessant à l'histoire. Cet intérêt se trouva accru par la collaboration qui s'était établie entre Albert Faucher, ancien élève de H. Innis, et l'économiste Maurice Lamontagne. Ces rapports entre historiens et spécialistes des sciences sociales

21 Voir RHAF (1957): 43-438 et (1961): 455-460; «La mentalité et l'outillage économique du paysan canadien en 1760: à propos d'un document sur l'encan», dans BRH (1956): 133-139.

22 Publié aux Presses de l'Université Laval.

23 «Le mouvement des prix agricoles dans la province de Québec, 1760-1851», «Les rendements agricoles dans les seigneuries et les cantons du Québec, 1700-1850», "Le régime seigneurial dans le Québec, 1760-1854», dans C. Galarneau et E. Lavoie (éd.), France et Canada français du XVI ${ }^{e}$ au XX $X^{e}$ siècles (Québec, PUL, 1966), 35-48, 81-120, 159-176. Le texte sur les prix agricoles a reçu son complément dans un texte que nous avons récemment publié en collaboration avec J. Hamelin et R. Chabot, "Les prix agricoles dans les villes et les campagnes du Québec d'avant 1850: aperçus quantitatifs», dans Histoire sociale (1982): 83-127.

24 Histoire économique du Québec, 1851-1896 (Montréal, Fides, 1971).

25 A. Dubuc, "Problems in the Study of the Stratification of the Canadian Society from 1760 to $1840 »$, dans RASHC (1965): 13-29.

26 J.-C. Falardeau (éd.), Essais sur le Québec contemporain (Québec, PUL, 1953), 260 p.; P.-E. Trudeau, La grève de l'amiante (Montréal, Éditions Cité Libre, 1956), 430 p. 
dans lesquels nous avons été impliqué à l'époque, se sont continués même lorsque, au début des années 1960, la typologie de la Folk Society ${ }^{27}$ connut une certaine disgrâce par suite de la progression de la pensée néo-nationaliste aussi bien dans les sciences sociales que parmi les historiens. Un texte de Jacques Dofny et de Marcel Rioux, publié en 1962 dans la Revue française de sociologie, est d'autant plus significatif à cet égard que Rioux avait été celui qui, parmi les Canadiens français, avait le plus contribué à donner de la crédibilité au modèle de Redfield. Leur texte qui mettait de l'avant le concept de la classe ethnique appelé à un bel avenir s'appuyait sur un arrière-plan historique inspiré par les écrits de l'École historique de Montréal dont les interprétations pouvaient maintenant être étoffées par les travaux des théoriciens de la décolonisation.

C'est dans cette conjoncture marquée par la montée du mouvement indépendantiste, par l'émergence d'un courant d'histoire sociale axé sur la tradition des Annales et par l'intérêt croissant des sociologues, des économistes et même des démographes pour l'histoire, que parut en 1966 notre Histoire économique et sociale du Québec, 1760-1850. Comme ce livre était plutôt dense et, peut-être, difficile à digérer, la réaction des lecteurs ne fut pas immédiate. Mais, une fois la première série de comptes rendus passée, elle se précisa et, finalement, prit la forme d'une remise en question radicale et persistante de la part d'un ancien élève de Maurice Séguin, Jean-Pierre Wallot, qui travaillait depuis nombre d'années sur la première décennie du XIX ${ }^{\mathrm{e}}$ siècle et qui lia désormais son sort à un économiste, Gilles Paquet, ancien élève de Albert Faucher, qui avait toujours, on le sait, manifesté un vif intérêt actif pour l'histoire. Cette remise en question se fit sur un point qui leur parut essentiel à l'argumentation du livre: celui de l'origine de la crise agricole bas-canadienne. Dans une série d'articles inaugurée en 1967 et poursuivie de plusieurs façons mais avec diligence dans plusieurs revues, dans des livres, dont Économie québécoise publié en 1969, et jusqu'à tout récemment encore par un texte sur les dîmes ${ }^{28}$, les auteurs étendirent la discussion bien au-delà de la crise agricole. En plus de poser le problème des méthodes au niveau de la théorie, de la modéli-

$27 \quad$ M. Rioux et Y. Martin (ed.), French Canadian Society (Toronto, McClelland and Stewart, 1964), 405 p. Collection d'articles qui permettent de saisir l'évolution de ce courant historiographique, et comprend, entre autres, un important texte de Rioux et Dofny (p. 307-319) ainsi qu'un texte de A. Faucher et M. Lamontagne (p. 256-271).

${ }_{28}$ G. Paquet et J.-P. Wallot, «Aperçus sur le commerce international et les prix domestiques dans le Bas-Canada (1793-1812)», RHAF (1967): 457-473; Patronage et pouvoir dans le Bas-Canada (1794-1812). Un essai d'économie historique (Montréal, PUQ, 1973), 182 p. (Reprise de quatre articles parus dans la RHAF, 1969-1970); «Le Bas-Canada au début du XIX ${ }^{\mathrm{e}}$ siècle: une hypothèse», RHAF (1971): 39-63; «Crise agricole et tensions socio-ethniques dans le Bas-Canada, 1802-1812», RHAF (1972): 185-236; «The Agricultural Crisis in Lower Canada, 1802-1812: mise au point. A Response to T.G.A. Le Goff», CHR (1975): 134-168; «Les inventaires après décès à Montréal au tournant du XIX ${ }^{\mathrm{e}}$ siècle: préliminaires à une analyse», RHAF (1976): 163-222; «Structures sociales et niveaux de richesse dans les campagnes du Québec, 1792-1812», dans Bulletin d' histoire de la culture matérielle, Musée national de l'homme (printemps 1981): 25-45. 
sation et des procédés quantitatifs, ils firent tout l'itinéraire qui allait de l'économique aux structures sociales ${ }^{29}$. Comme l'enjeu de ce débat n'intéressait pas seulement les parties en cause, des historiens, tel T. Le Goff ${ }^{30}$, des économistes, tels McCallum ${ }^{31}$, McGinnis ${ }^{32}$, Lewis et Armstrong ${ }^{33}$, des géographes, tel Courville ${ }^{34}$, et même des sociologues s'en mêlèrent, de sorte que cet échange à voix multiples me semble vraiment significatif de l'apparition d'un nouveau contexte pluridisciplinaire dans lequel se développe une historiographie qui n'appartient plus en exclusivité aux historiens.

Ceci dit, le schéma que proposent Wallot et Paquet pour arriver à définir les rapports entre les groupes sociaux au début du XIX ${ }^{\mathrm{e}}$ siècle repose sur trois concepts: celui d'économie atlantique, celui de modernisation et celui de blocage, et même un quatrième: celui de la frontière ${ }^{35}$. En effet, il leur semble que si les choses s'étaient déroulées jusque-là normalement, la société bas-canadienne aurait poursuivi jusqu'au bout sa marche vers la modernité: c'est-à-dire vers la révolution bourgeoise. Mais, disent-ils, certains blocages comme le système colonial et quelques autres également attribuables aux anglophones comme la division de la bourgeoisie par l'ethnie ont contribué à désamorcer «le procès révolutionnaire». Ce modèle d'analyse qui insiste exclusivement sur les contraintes exogènes pour expliquer l'échec de la course victorieuse vers la modernité présente des similitudes frappantes avec la thèse néonationaliste originelle. Il s'en distingue néanmoins en ce sens que l'intervention des forces économiques, sans être bien évidente, est prévue dans le mécanisme explicatif et que, de cette façon, il existe une possibilité qu'on ne soit pas à cent pour cent en présence d'un simple conflit de nationalités. La chose est d'autant plus plausible qu'en arrière-plan à cette thèse modernisante se trouve, sans que cela soit établi, une autre perspective non moins modernisante: celle de la frontière ${ }^{36}$. Ainsi, der-

29 Pour nos réponses, voir F. Ouellet, «L'agriculture bas-canadienne vue à travers les dîmes et la rente en nature», Histoire sociale (1971): 5-45; «La sauvegarde des patrimoines dans le district de Québec durant la première moitié du XIX ${ }^{\mathrm{e}}$ siècle», RHAF (1972): 319-375; «Le mythe de l'habitant» sensible au marché». Commentaires sur la controverse Le Goff - Wallot et Paquet», Recherches sociographiques (1976): 115-132.

30 T.J.A. Le Goff, «The Agricultural Crisis in Lower Canada, 1802-1812. A Review of a Controversy», CHR (1975): 134-168.

31 J. McCallum, Unequal Beginnings. Agriculture and Economic Development in Quebec and Ontario Until 1870 (Toronto, UTP, 1980).

32 R.M. McGinnis, «A Reconsideration of the State of Agricultures in Lower Canada in the First Half of the Nineteenth Century», in Canadian Papers in Rural History, 3 (1982): 15-77.

33 R. Armstrong, «The Efficiency of Quebec Farmers in 1851», à paraître.

34 S. Courville, «La crise agricole du Bas-Canada. Éléments d'une réflexion géographique», Cahiers de géographie du Québec (1980): 193-224, 385-428.

35 Voir «Groupes sociaux et pouvoir: le cas canadien au début du XIX ${ }^{\mathrm{e}}$ siècle», RHAF (1974): 509-564.

36 J.-P. Wallot, «Le régime seigneurial et son abolition au Canada», dans Un Québec qui bougeait. Trame socio-politique au tournant du XIX $X^{e}$ siècle (Montréal, Boréal Express, 1973), 225-253. Nous avons discuté de cette mise en perspective dans «La formation d'une société dans la vallée du Saint-Laurent...», CHR (1981): 407-450. 
rière tout cela, il y avait déjà tout un appareil d'Ancien Régime désintégré sous l'effet de l'environnement et, c'est pourquoi, il n'y avait plus de possibilité qu'un procès révolutionnaire naisse de l'intérieur même de la société. Il est évident, malgré les complications apparentes de l'analyse et les finesses du langage, que la «gentilité bureaucratique et militaire, la noblesse seigneuriale, la grande bourgeoisie d'affaires, les moyenne et petite bourgeoisies, les classes populaires urbaines, les classes populaires rurales et le clergé», groupes dont parlent Wallot et Paquet, ne sont plus, tels que définis et agencés, disponibles pour une lutte dont l'enjeu serait soit une révolution bourgeoise, soit une révolution populaire. Le refus de lier même faiblement la naissance du nationalisme à la transformation des structures économiques (dont la crise agricole n'était pourtant qu'un aspect) et à l'émergence d'une crise démographique, est, à notre avis, la conséquence normale de ce schéma interprétatif. Dans ce contexte, pour cette raison et pour d'autres, les résultats extraordinairement et universellement positifs de leur enquête dans les inventaires après décès, me paraissent sujets à caution ${ }^{37}$. Finalement, malgré tout ce brouhaha, on se retrouve encore devant une simple lutte pour le patronage et le pouvoir et devant un simple conflit de nationalités. Ici, l'histoire sociale, pas plus que l'histoire économique, ne trouve vraiment son compte. On est toujours en présence de la conception d'une société homogène, stratifiée mais harmonieuse, soumise aux seules menaces de l'extérieur.

\section{Le débat sur les insurrections de 1837-38: les rapports entre le national et le social}

Le troisième débat auquel j'ai fait allusion dans cette tentative pour situer les recherches sur les classes dominantes et qui est relié aux deux autres, porte sur la signification, dans la perspective nationale et sociale, des insurrections de 1837-38. En effet, de Garneau à Filteau, les historiens canadiens-français sans exception ont précisément, par leur

37 Voir la RHAF (1976): 163-222 et Bulletin d'histoire de la Culture matérielle, Musée national de l'homme (printemps 1981): 25-45. À notre avis, c'est la méthodologie de ces textes qui est en défaut. L'échantillon ici utilisé ne semble pas avoir été construit avec rigueur en se préoccupant suffisamment du caractère représentatif des résultats obtenus. Ainsi, la décision de comparer une conjoncture basse (1792-96) et une conjoncture haute (1807-1812) n'a pas fait l'objet d'une justification pas davantage que celle d'accepter comme significatifs des chiffres qui comportent des écarts plutôt aberrants. Ainsi, si on en croit les données tirées de ces inventaires, l'étendue moyenne de la propriété foncière serait passée dans la ville de Québec de 1,5 arpents en 1792-96 à 18,0 arpents en 1807-12 et à Montréal, pendant les mêmes années, de 54 arpents à 387 (voir Bulletin de la culture matérielle, p. 30s.). Ces chiffres sont d'autant plus étonnants que d'habitude les emplacements urbains ne totalisent dans les meilleures circonstances que des fractions d'arpents. C'est certainement encore le cas en 1831: l'emplacement moyen, calculé à partir du recensement, ne dépasse pas à Québec 0,28 d'arpent et à Montréal 0,44 . Les chiffres extraits des inventaires après décès concernant les animaux de la ferme présentent les mêmes anomalies: ils ne cadrent certes pas avec les moyennes calculées à partir des données des recensements de 1765,1784 et 1831 . En vérité, G. Paquet et J.-P. Wallot n'ont pas réussi à démontrer quelles catégories de propriétaires étaient représentées dans les inventaires après décès retenus dans leur échantillon. 
interprétation, contribué, pour utiliser l'expression de J.-Pierre Wallot et $\mathrm{G}$. Paquet, à désamorcer le procès révolutionnaire. Car, au lendemain de l'échec de 1837-38, le sentiment prévalait parmi les dirigeants de la société (en particulier le clergé) d'avoir échappé à un immense danger ${ }^{38}$. Plus répandu après la défaite, autant chez les acteurs du drame que chez leurs adversaires, existait la volonté qu'il fallait, dans la mesure du possible, oublier et, surtout, chasser le désir de poser le problème des responsabilités des personnes impliquées. Nier jusqu'à l'existence même d'un projet révolutionnaire parut la solution la plus souhaitable, ou en tout cas, la moins douloureuse. La thèse justificatrice de Papineau selon laquelle personne n'avait prémédité la lutte armée et selon laquelle les patriotes n'avaient fait que résister en masse à l'arrestation des chefs, sembla parfaitement acceptable en l'occurrence et ne fit l'objet d'aucun effort de vérification même après la querelle Papineau-LaFontaine.

Cette détermination d'oublier et de nier afin que les choses ne recommencent pas fut renforcée non seulement au XIX ${ }^{\mathrm{e}}$ siècle par les dangers que représentèrent en ce sens le mouvement annexionniste, celui de l'Institut canadien et le rougisme, mais au $\mathrm{XX}^{\mathrm{e}}$ siècle par les craintes qu'inspirèrent en certains milieux l'industrialisation et l'urbanisation. Ce furent ces réactions que traduisirent, pour une bonne part, les historiens. Benjamin Sulte disait que les Canadiens, à l'époque des insurrections, préféraient la «discussion paisible» ${ }^{39}$. Lui aussi d'ailleurs. L'historiographie de la lutte pour la survivance a ainsi fait sa part pour désamorcer les insurrections en tant que précédent révolutionnaire de plusieurs façons: soit en les condamnant directement, soit en minimisant leur portée, soit, ce qui revient au même, en insistant sur la sincérité de certains de leurs acteurs. Dans son Histoire du Canada depuis la découverte ${ }^{40}$, Groulx va jusqu'à proposer à ses lecteurs d'éliminer même de leur vocabulaire les mots rébellions et insurrections pour les remplacer par une terminologie moins surchargée de sens: mutineries ou soulèvements. Pourtant, il arrivera à Groulx un peu plus tard dans son texte, se référant sans doute à un impossible rêve d'indépendance, de mentionner «en toute sa gravité et profondeur l'émoi du pays ${ }^{41}$. En somme, admettre parfois l'existence d'une situation révolutionnaire mais nier celle d'un projet révolutionnaire, dénoncer les idées démocratiques ou libérales pour les attribuer à une minorité non représentative, irresponsable ou perdue dans l'univers des idées ou, encore, parler des bonnes intentions de certains individus et, enfin, insister démesurément sur les limites géographiques du mouvement

\footnotetext{
38 Nous avons étudié, dans un texte à paraître, cette question: «La tradition révolutionnaire au Canada. À propos de l'historiographie des insurrections de 1837-38 dans le Bas-Canada».

39 Histoire des Canadiens français, 1608-1880 (Montréal, Société de publication historique du Canada, 1884), 121. III: 224

40 Histoire du Canada français depuis la découverte (Montréal, L'Action nationale, 1952),

41 L. Groulx, Ibid.: 229.
} 
patriote ou sur celles de la participation populaire, tout cela fit partie d'une tradition plus que séculaire qui eut pour conséquence soit d'éliminer ces événements de l'histoire en tant que précédent dangereux ou de les réintégrer en les désamorçant. Le rôle des classes dirigeantes dans ce cadre dominé par la question nationale se définit en fonction de la sauvegarde de la société traditionnelle et de la lutte plus ou moins paisible pour la conquête paisible des libertés parlementaires.

C'est donc à partir de 1950 que les obstacles à la mise en place d'une interprétation qui attribuait aux rébellions certains caractères d'un mouvement révolutionnaire commencèrent à être levés. L'École néonationaliste fit un pas considérable dans cette direction. Sa définition même de la nation liait le caractère catastrophique de la conquête à une conception de la décapitation sociale qui faisait émerger l'objectif de l'indépendance comme une conséquence normale, sinon inévitable, des événements de 1760 . «Ce mouvement séparatiste canadien-français de 1763 à 1837, disait Maurice Séguin, est naturel. Ce n'est pas la politique anglaise qui l'a créé. Elle n'a fait que l'intensifier et le cultiver.» ${ }^{42}$ La seule chose qui, à long terme, pouvait reporter la réalisation de ce rêve à plus tard était, chez les Canadiens français, «la crainte de la puissance américaine» ${ }^{43}$. C'est dans cette perspective de longue durée et en fonction de ces postulats que les premiers néo-nationalistes analysèrent brièvement les insurrections de 1837-38. Selon eux, l'objectif des Patriotes était de n'atteindre l'indépendance que «dans 50 ou 100 ans»; mais, en attendant ce grand jour, les Patriotes ne visaient qu'à la conquête d'un statut de quasi-indépendance acquis par des moyens pacifiques et non par la force. Mais, même cet objectif devenait irréalisable parce qu'il allait à l'encontre des visées d'une autre nation occupant le même territoire: la nation Canadian qui, se sentant menacée, aurait alors pris l'initiative de la confrontation. Il s'agissait donc essentiellement d'une lutte de «nation contre nation à travers un conflit constitutionnel» qui dégénéra bientôt, par l'entremise des éléments avancés des deux collectivités, en un affrontement armé ${ }^{44}$. À cet égard, le mot de la fin appartient à Michel Brunet: «L'agitation, dit-il, et la démagogie des extrémistes conduisirent au feu de 1837-38. Papineau et ses partisans n'avaient pas tenu compte d'un fait essentiel: le parti canadien n'était pas libre d'adopter un programme politique et économique contraire aux intérêts fondamentaux de la colonisation anglaise au Canada. ${ }^{45}$ Ainsi évaluées, comme prématurées et liées aux contingences des visées individuelles, les insurrections ne pouvaient encore

\footnotetext{
${ }^{42}$ M. Séguin, L'idée d'indépendance au Québec. Genèse et historique (T.-Rivières, Boréal Express, 1968), 17 .

43 M. Séguin, Ibid., 21.

44 M. Séguin, Ibid., 33.

45 M. Brunet, La Présence anglaise et les Canadiens. Études sur l'histoire de la pensée des deux Canadas (Montréal, Beauchemin, 1964), 152.
} 
constituer le grand précédent qu'elles deviendront plus tard pour les indépendantistes québécois et, il faut le dire, pour les historiens.

En tout cela, c'est encore la question nationale qui seule compte et commande le comportement des classes dominantes: la petite bourgeoisie canadienne-française et le clergé, d'une part, la minorité anglophone marchande et bureaucratique, d'autre part. Pour Brunet et Séguin, tous les problèmes de la société canadienne-française découlaient d'une source extérieure unique: le phénomène de la colonisation britannique engagée par la conquête de 1760 dont les conséquences à long terme avaient été d'autant plus néfastes que cette société avait en quelque sorte réalisé sa révolution bourgeoise dès l'époque de la NouvelleFrance. S'il était vrai, par exemple, que les anglophones étaient les seuls et véritables responsables des déviations subséquentes de l'institution seigneuriale, il était donc normal que les historiens néonationalistes à la suite de la petite bourgeoisie patriote elle-même n'aient pas accordé d'importance à la liquidation de l'Ancien Régime social en tant que source endogène d'exploitation de la population coloniale. Mais ceci n'élimine pas le fait qu'il était devenu une source exogène d'exploitation et de domination et que, sur ce plan, l'attitude pro-seigneuriale des Patriotes se justifie mal.

De notre côté, nous avons toujours rejeté cette prise en charge de l'interprétation des rébellions par le seul biais du national. Au départ, dans un de nos premiers articles publié en 1954, nous décrivions le mouvement patriote comme étant simplement l'expression en terre nordaméricaine des mouvements nationalistes et libéraux de l'époque ${ }^{46}$. Mais, bientôt, il nous apparut que non seulement les rapports entre nationalisme et libéralisme étaient plus complexes mais que les racines de ce mouvement étaient également plus diverses et enchevêtrées lorsqu'elles étaient analysées avec un certain sens de la longue durée sous l'angle économique et social auquel nous allions ajouter plus tard la dimension démographique ${ }^{47}$.

C'est pourquoi avec le temps, et en utilisant le cadre quantitatif le plus élaboré possible, nous avons été amené à voir se constituer, ainsi que nous l'avons déjà dit, une structure socio-économique et démographique qui, nous le pensons, devait demeurer à peu près la même du début du XVIII ${ }^{\mathrm{e}}$ siècle au début du siècle suivant, alors que s'amorce le premier changement majeur dans ces structures avant l'industrialisation. Le facteur qui, au départ, commande cette mutation et déclenche

\footnotetext{
46 F. Ouellet, «Causes économiques et origines intellectelles de la révolution de 1837: Autour du dernier livre du chanoine Groulx», Hermès, (1954): 22-35.

47 F. Ouellet, «Papineau dans la révolution de 1837-38», RASHC (1958): 13-34; Histoire économique et sociale du Québec, 1760-1850 (Montréal, Fides, 1966), 640 p.; «Les insurrections de 1837-38 comme phénomène social», Histoire sociale (1968): 54-82; Le Bas-Canada. Changements structuraux et crise, 1791-1840 (Ottawa, EUO, 1976), 539 p.
} 
une crise globale, qui déboucha finalement sur les insurrections, est l'économique. Comme seconde composante de cette crise se trouve le facteur démographique, dont l'impact se fait de plus en plus considérable avec le temps. À mesure que cette crise économique et démographique se propage et s'approfondit d'une façon inégale selon les régions, les rapports entre les classes sociales se modifient et confèrent à la lutte tous les caractères d'une lutte de classes compliquée par les changements d'équilibres entre les groupes ethniques. Ainsi, le jeu des forces économiques et démographiques combinées, dont l'immigration massive des Britanniques devient une composante capitale, est à l'origine du développement d'un prolétariat dans les campagnes et de disparités économiques accrues entre les ethnies. La montée du nationalisme dans la société bas-canadienne, comme il se doit en l'occurrence, s'en trouve stimulée. À l'époque de notre Histoire économique et sociale du Québec, nous avons eu tendance à représenter le mouvement patriote des années 1834-38 sous les couleurs d'un mouvement nationaliste, dominé par la petite bourgeoisie, habité par l'idée d'indépendance, utilisant un discours politique libéral, démocratique, voire républicain, mais foncièrement voué dans l'ensemble à la défense de la structure sociale d'Ancien Régime. Depuis ce temps, nous avons distingué à l'intérieur de ce mouvement à tendance conservatrice, sinon réactionnaire, une aile gauche minoritaire qui sans doute visait à l'indépendance mais qui voyait en celle-ci la condition nécessaire à une révolution sociale. Dans ces circonstances, c'est le développement d'un conflit entre ces deux groupes à propos des objectifs sociaux de leur mouvement, qui aurait joué le plus fortement sur l'issue de la lutte armée. Cette interprétation met en évidence l'existence d'une situation révolutionnaire porteuse de projets et de stratégies du même ordre par des groupes qui disposent d'appuis étendus dans les milieux populaires; elle repose en outre sur une exploration, par le biais de l'Ancien Régime social en tant que source interne de la crise, des rapports entre le national et le social.

Dès la fin des années 1960, l'historiographie néo-nationaliste fut incitée à se transformer, d'abord sous l'effet de la poussée nationaliste qui donna un relief incomparable à l'idée d'indépendance, puis sous l'influence du développement, dans la société et les milieux intellectuels, d'une sensibilité beaucoup plus attentive à la dimension sociale des problèmes. Il va sans dire que cette situation devait favoriser l'intégration pleine et entière des rébellions dans le discours historien, en tant que précédent révolutionnaire annonciateur du grand événement. C'est un fait dont témoignent, entre autres, les pèlerinages annuels à St-Denis. C'est également un fait qu'illustrent deux courants qui me paraissent issus de ce changement: un courant nationaliste socio-culturel et un courant marxiste.

En effet, depuis 1960, l'historiographie a eu tendance au plan international à délaisser peu à peu les perspectives socio-économiques 
en faveur d'éclairages socio-culturels. Dans le premier temps de cette évolution, l'histoire sociale, au lieu de s'appuyer comme autrefois sur l'économique, a davantage misé sur les enseignements de la démographie pour, un peu plus tard, se laisser attirer par l'anthropologie. Cette utilisation des concepts anthropologiques née d'une attention plus subtile aux faits culturels est aussi fréquente dans les travaux inspirés par les mouvements de décolonisation. De cela, on trouve la trace évidente dans le livre plutôt curieux de Denis Monière, Les idéologies au Québec... ${ }^{48}$, paru en 1977 , qui fait en même temps appel à la théorie du développement du sous-développement, au concept de classe ethnique, à celui du centre-périphérie, à des auteurs comme Fanon, Mao, et Marx, et qui réussit à marier tout cela avec les idées de Groulx, de L. Dechêne et de Michel Brunet. Quoi qu'il en soit, le recours à l'ensemble de ces concepts anthropologiques ou à l'un ou l'autre d'entre eux a permis d'apporter une dimension sociale substantielle à la pensée néo-nationaliste.

Un livre de synthèse qui rend bien compte de cette orientation est celui de Jean-Claude Robert, Du Canada français au Québec libre. Histoire d'un mouvement indépendantiste ${ }^{49}$, publié en 1975. L'auteur avait auparavant rédigé une thèse qui faisait apparaître au coeur même des activités de Barthélemy Joliette, seigneur de Lavaltrie ${ }^{50}$, le rôle des Canadiens français dans le domaine du capitalisme foncier et les retombées de ce capital sur le développement de la petite industrie. Quelques années plus tard, en collaboration avec Paul-André Linteau qui explorait le même problème dans son étude sur la ville de Maisonneuve, il faisait émerger la présence plus que significative des francophones dans le secteur de la propriété foncière dans la ville de Montréal ${ }^{51}$.

Ces deux historiens qui étaient alors engagés dans des recherches sur l'histoire urbaine, n'étaient pas les seuls à travailler dans cette direction qui pouvait servir à démontrer l'existence d'une stratégie économique chez les Patriotes et, d'une façon plus générale, à éclairer la question du faible poids des Canadiens français dans le monde capitaliste. En effet, certains écrits de André Garon ${ }^{52}$ et les recherches de Normand Séguin ${ }^{53}$ allaient dans le même sens.

La perspective de base du livre de Robert découle sans aucun doute du modèle néo-nationaliste mais il est également clair que son auteur

\footnotetext{
48 Le développement des idéologies au Québec des origines à nos jours (Montréal, Éditions Québec-Amérique, 1977), 381 p.

49 Publié chez Flanmarion dans la collection Histoire vivante, 323 p.

50 «Un seigneur entrepreneur, Barthelémy Joliette, et la fondation du village de l'industrie», RHAF (1972): 319-374.

51 «Propriété foncière et société à Montréal: une hypothèse», RHAF (1974): 45-66.

52 Histoire du Québec (St-Hyacinthe, Privat, 1976), 231-345. Du même auteur, voir: «La fonction politique et sociale des chambres hautes canadiennes, 1791-1841», Histoire sociale (1970): 66-88.

La conquête du sol au XIX ${ }^{e}$ siècle (Québec, Boréal Express, 1977), 295 p.
} 
était sensible à la dimension économique et sociale des problèmes. Aussi, son analyse des conséquences à moyen et à long terme de la conquête est-elle beaucoup plus nuancée que celle des premiers néo-nationalistes. Cette attitude correspond d'ailleurs à celle d'un certain nombre de jeunes chercheurs qui ont écrit depuis les années 1970 sur le rôle de la bourgeoisie canadienne-française à cette époque et plus tard. Je pense en particulier aux articles de José Igartua ${ }^{54}$ sur les commerçants de pelleteries, de Louis Michel ${ }^{55}$ sur les marchands de campagne et à un texte encore plus récent de Georges Bervin ${ }^{56}$ sur le contexte dans lequel évoluent les bourgeois de Québec dans les années 1820-1830.

Il en est de même de la mise en place de l'arrière-plan des rébellions qui, même si Robert est forcé de faire le plus court possible, fait appel à une vision assez complexe des facteurs et des forces en présence, dont les classes sociales. Mais ce n'est pas d'abord à ce niveau que se situe son explication principale; celle-ci s'appuie plutôt sur des concepts anthropologiques, comme celui d'acculturation, et elle débouche en fin de compte sur l'idée d'un affrontement entre deux sous-cultures. Il est évident que cet intérêt pour le culturel est appelé dans l'avenir à prendre de l'ampleur et à se manifester de différentes façons parmi les historiens. Je pense ici à l'histoire du Québec de S. Trofimenkoff ${ }^{57}$, qui propose sa propre version féministe d'une histoire intellectuelle et culturelle.

Tenant compte de tout cela, on peut rattacher, en tout ou en partie, les études particulières parues depuis 1970 (qu'il s'agisse de livres ou d'articles de revues) sur les classes dominantes à l'une ou l'autre de ces tendances. Je songe en particulier à certains travaux de Pierre Tousignant ${ }^{58}$ sur le rôle actif de la bourgeoisie canadienne-française dans le mouvement pour l'obtention des institutions parlementaires, au livre de Richard Chabot sur Le curé de campagne... ${ }^{59}$ (1975), à celui de Gérald Tulchinski, The River Barons ${ }^{60}$ (1977), aux textes de B. Tunis

\footnotetext{
54 «A Change in Climate. The Conquest and the Merchants of Montreal», CHAAR (1974): 115-135; «The Merchants of Montreal at the Conquest: Socio-economic Profile», Histoire sociale (1975): 275-294: «Le comportement démographique des marchands de Montréal vers 1760», RHAF (1979): 427-447.

55 Un marchand rural en Nouvelle-France: F.-A. Bailly de Messein, 1709-1771», RHAF (1979): 215-263; «Le livre de compte de Gaspard Massue, marchand à Varennes (1784-1792)», Histoire sociale (1980): 369-398.

56 «Aperçu sur le commerce et le crédit à Québec, 1820-1830», RHAF (1983): 527-553; «Environnement matériel et activités économiques des conseillers exécutifs et législatifs à Québec, 1810-1830», Bulletin d' histoire de la culture matérielle, Musée national de l'homme (printemps 1981): 45-63.

57 The Dream of Nation. A Social and Intellectual History of Quebec (Toronto, Macmillan, 1982), $344 \mathrm{p}$.

58 «Problématique pour une nouvelle approche de la constitution de 1791», RHAF (1973): 181-234; «La première campagne électorale des Canadiens en 1792», Histoire sociale (1975): $120-148$.

59 Le curé de campagne et la contestation locale au Québec de 1791 aux troubles de 183738 (Montréal, HMH, 1975).

60 Publié par les Presses de l'Université de Toronto (1975), 310 p.
} 
et de J. Bernier sur la profession médicale, aux volumes de Claude Galarneau $^{61}$ sur La France et l'opinion canadienne (1970), et sur les Collèges classiques (1978) et, enfin, à celui de C. Baribeau, sur la Petite-Nation $^{62}$. Je pense aussi à la diversité des préoccupations qui ont présidé à la rédaction des biographies préparées à l'intention du Dictionnaire biographique du Canada, dont plusieurs volumes intéressent au premier chef les personnages influents des années 1760 à 1840. Mais il est un courant qui, depuis plusieurs années, a pris une importance assez considérable et qui a acquis de plus en plus de signification dans les trois débats qui constituent la base de cet exposé. Il s'agit de l'historiographie marxiste.

Cette historiographie marxiste avait déjà vers 1970 ses traditions. En effet, même s'il avait plutôt prêché dans le désert depuis 1937 au moins, Stanley Ryerson avait porté le flambeau d'une interprétation marxiste de l'histoire canadienne et québécoise appuyée sur la théorie de la transition du féodalisme au capitalisme. À cette date, dans son livre: 1837. The Birth of Canadian Democracy ${ }^{63}$, il pouvait reprocher à la bourgeoisie nationale canadienne, cette grande bénéficiaire, à ses yeux, de l'oeuvre révolutionnaire de 1837-38, non seulement de faire le silence sur ces événements, de les avoir voués à l'oubli, mais de signifier à tous, en agissant ainsi, sa haine de la démocratie et du progrès. A cette époque, Ryerson avait plutôt tendance, comme cela sera le cas de bien des intellectuels québécois durant les années 1950, à se méfier du nationalisme en tant que force conservatrice. On comprend alors que dans ce premier livre, il n'ait pas tellement accordé d'importance à la question de la décapitation sociale qui, selon lui, signifiait seulement le remplacement d'une classe dirigeante seigneuriale francophone par une classe à peu près équivalente d'anglophones ${ }^{64}$. Cette subordination absolue du national au social devait devenir de moins en moins rigide par la suite. Dans son livre de 1944, French Canada ${ }^{65}$, il continuera au sujet des insurrections de parler de révolution populaire, démocratique, anti-féodale et anti-coloniale mais on sent déjà qu'il réféchit sur la véritable dimension de la nation, puisqu'il mettait davantage l'accent sur le caractère national de la lutte dans le Bas-Canada, sans pourtant vraiment insister sur les divisions qui existaient dans le camp patriote sur la question seigneuriale. En 1960, un nouveau pas était fait et il ira jusqu'à décrire le parti canadien de Pierre Bédard comme un «national-

\footnotetext{
61 Le premier publié aux PUL et le second chez Fides.

62 La seigneurie de la Petite-Nation. Le rôle économique et social du seigneur (Hull, Asticou, 1983), 165 p. Voir aussi R.C. Harris, «Of Poverty and Helplessness in Petite-Nation», CHR (1971): 23-49.

63 (Toronto, Francis White Publishers, 1937), 136 p.

64 S.B. Ryerson, ibid., 31.

65 (Toronto, Progress Books, 1944), 248 p.
} 
démocratic» ${ }^{66}$ party. Huit ans plus tard, dans Unequal Union ${ }^{67}$, sa pensée avait évolué à tel point qu'on pourrait croire qu'il était sur le point d'accepter le concept de classe ethnique, tellement il avait incorporé d'éléments de l'interprétation néo-nationaliste dans son discours. Dans l'édition française de ce livre paru en 1972 sous le titre Le capitalisme et la confédération ${ }^{68}$, il expliquait en avant-propos que de «nos jours, on assiste à un certain rapprochement sinon à une convergence des nationalismes qui se veulent à gauche et de syndicalismes à la recherche des voies de l'action politique». Non seulement insistait-il sur les traits industriels du capitalisme qui se développait au début du XIX ${ }^{\mathrm{e}}$ siècle autour de l'exploitation forestière, mais toujours dans le but de mettre en évidence les arrière-plans des rébellions, il notait l'apparition, chez les Canadiens français, d'un embryon de bourgeoisie industrielle et ne mentionnait même pas les conflits qui existaient chez les Patriotes à propos des institutions d'Ancien Régime.

En somme, d'une étape à l'autre, en restant toujours fidèle à la théorie de la transition mais en révisant constamment ses conceptions des rapports entre le national et le social, il avait mis au point une interprétation des insurrections qui avait des chances d'exercer une influence sur nombre de Québécois nationalistes attirés par le marxisme. Curieusement, parmi les francophones nationalistes touchés par la pensée de Ryerson, Gilles Bourque fut celui qui représenta davantage pendant nombre d'années cette historiographie marxiste mais, il faut le noter, en accomplissant un itinéraire inverse de celui de Ryerson.

Dans Classes sociales et question nationale ${ }^{69}$, en 1970, Bourque était parti du concept de classe ethnique et, en utilisant jusqu'à un certain point les écrits des néo-nationalistes et les écrits à caractère socioéconomique, il était parvenu à donner aux insurrections une signification nationale, bourgeoise, capitaliste «à vocation industrielle», populaire et démocratique qui inscrivait les événements révolutionnaires dans le sens d'une histoire à faire. Mais, au cours des années 1970, les choses évoluèrent rapidement et Bourque commença à prendre ses distances par rapport à un modèle qui valorisait tellement la question nationale et ne semblait plus répondre à ses besoins. Son livre, Le Québec. La question nationale $e^{70}$ (1979), publié avec Anne Légaré, est un retour à une théorie qui, en soi, garantissait au social sa suprématie. De cette façon, Bourque aboutissait à une nouvelle conception de la société à l'époque de la Nouvelle-France, une société dans laquelle l'élément féodal dominait et à une nouvelle version des rébellions de 1837-38

\footnotetext{
66 The Founding of Canada. Beginnings to 1815 (Toronto, Progress Books, 1960), 278s.

67 (Toronto, Progress Books, 1968), 23, en particulier.

68 Avec sous-titre: Aux Sources du Conflit Canada-Québec (1760-1873) (Montréal, Edition Parti-Pris, 1972), 13

69 (Montréal, Éditions Parti-Pris, 1970), 350 p.

70 (Paris, PCM, 1979), 232 p.
} 
centrée sur les contradictions de la petite bourgeoisie, laquelle disaitil, était incapable de véhiculer autre chose qu'un projet national sans projet social le moindrement clair. On peut se demander si, dans le contexte actuel, cette orientation, qui traduit les déceptions de bien des éléments de gauche à propos du parti québécois, a des chances de susciter beaucoup d'adhésions. Il est certain en tout cas, qu'elle ne semble pas avoir rallié Gérald Bernier et Daniel Salée ${ }^{71}$ qui, eux aussi, sur le thème de la transition, sont en voie de mettre au point une autre version marxiste plus attentive à la question nationale des transformations de la société québécoise au cours du siècle qui suit la conquête de 1760. Bien sûr, les modèles marxistes ont tous un caractère global. Comme tels, ils débouchent aussi bien sur l'étude des classes dominantes que sur celle des classes populaires. Il n'en reste pas moins que, jusqu'à ce jour, la recherche a surtout été orientée là où allaient davantage les sympathies: c'est-à-dire vers les paysans, la question seigneuriale et les artisans.

De tout cela se dégage l'idée que le présent est une des principales sources de nos certitudes et de nos interrogations. Comme tel, non seulement fournit-il des perspectives d'ensemble mais il nous incite à revoir certains dossiers ou à en ouvrir de nouveaux, de sorte que l'orientation prochaine de la recherche sera influencée par l'évolution même de la société. Il faut dire que les historiens ne sont pas à cet égard dans une position plus précaire que celle des spécialistes des autres sciences de l'homme. Peut-être sont-ils simplement devenus plus conscients qu'eux de l'existence de ces déterminismes qui infléchissent leur pratique dans un sens ou dans l'autre. À ce niveau, il n'est pas évident du tout que le mouvement récent et général vers le socio-culturel et les préoccupations nouvelles pour la culture des élites ou la culture populaire auront pour effet d'alimenter, bien qu'on aime toujours à parler de la culture des élites, de la culture populaire et de la culture de la classe ouvrière, l'intérêt qui, peu à peu, s'est développé pour une histoire attentive aux classes sociales. Si cela est vrai, notre penchant pour l'étude des classes dominantes et des classes dominées irait, pendant un bon moment encore, à l'encontre du courant et pourrait bien s'effriter.

Ceci dit, il faut bien admettre que ce chantier, même réduit aux enquêtes sur les classes dominantes, est bien grand comparé à nos modestes réalisations. Qu'il s'agisse des travaux sur les bourgeoisies, sur l'aristocratie ou sur le clergé, ce n'est pas le goût de généraliser qui fait défaut mais la capacité de s'engager dans des entreprises d'enver-

71 G. Bernier, «Sur quelques effets de la rupture structurelle engendrée par la conquête au Québec, 1760-1854», RHAF (1981): 69-97; G. Bernier et D. Salée, «Appropriation foncière et bourgeoisie marchande: éléments pour une analyse de l'économie marchande du Bas-Canada avant 1846», RHAF (1982): 163-195. 
gure combinant des perspectives larges et un énorme labeur empirique propice à la réflexion. Il y a trop de chercheurs dont l'effort consiste sans plus à conceptualiser et ne parvient pas à se porter plus loin, sur un terrain rocailleux où se fait à partir des sources originales le travail de vérification et d'analyse en profondeur. Pour briser ce goût de l'inachevé, il est urgent de multiplier les solides entreprises monographiques sans lesquelles le renouvellement de l'historiographie, au lieu de dépendre de la réflexion méthodologique pratiquée au contact des archives, est uniquement soumis au brassage idéologique. 